Journal of Patient-Centered

\title{
Dancing During Labor: Social Media Trend or Future Practice?
}

\author{
Caroline P. Toberna \\ Drew Horter \\ Kayla Heslin \\ Marie M. Forgie \\ Emily Malloy \\ Jessica J. F. Kram
}

Follow this and additional works at: https://aah.org/jpcrr

Part of the Alternative and Complementary Medicine Commons, Family Medicine Commons, Maternal and Child Health Commons, Maternal, Child Health and Neonatal Nursing Commons, Obstetrics and Gynecology Commons, and the Reproductive and Urinary Physiology Commons

\section{Recommended Citation}

Toberna CP, Horter D, Heslin K, Forgie MM, Malloy E, Kram JJ. Dancing during labor: social media trend or future practice? J Patient Cent Res Rev. 2020;7:213-7. doi: 10.17294/2330-0698.1723

Published quarterly by Midwest-based health system Advocate Aurora Health and indexed in PubMed Central, the Journal of Patient-Centered Research and Reviews (JPCRR) is an open access, peer-reviewed medical journal focused on disseminating scholarly works devoted to improving patient-centered care practices, health outcomes, and the patient experience. 


\title{
Dancing During Labor: Social Media Trend or Future Practice?
}

\author{
Caroline P. Toberna, BS, 1,2,3 Drew Horter, ${ }^{1,2,3}$ Kayla Heslin, MPH,, ${ }^{1,2,3}$ Marie M. Forgie, DO, ,4,6 \\ Emily Malloy, APNP, CNM,2,5,6 Jessica J. F. Kram, MPH²,3
}

${ }^{1}$ Aurora Research Institute, Aurora Health Care, Milwaukee, WI; ${ }^{2}$ Aurora UW Medical Group, Aurora Health Care, Milwaukee, WI; ${ }^{3}$ Center for Urban Population Health, Milwaukee, WI; ${ }^{4}$ Obstetrics and Gynecology, Aurora Sinai Medical Center, Milwaukee, WI; ${ }^{5}$ Midwifery and Wellness Center, Aurora Sinai Medical Center, Milwaukee, WI; ${ }^{6}$ Department of Obstetrics and Gynecology, University of Wisconsin School of Medicine and Public Health, Madison, WI

\begin{abstract}
Over the past 30 years, medical interventions in pregnancy and childbirth have increased. Some pregnant women seek less invasive and nonpharmacological options to manage discomforts during labor. While exercise during pregnancy is recommended, less is known about exercise, specifically dancing, during labor. While anecdotal evidence is supportive, little is known about the implications of exercise and dance during the first stage of labor for pain reduction and labor progression. Some movements common in dance, such as expanding hip circles that loosen and relax muscles of the pelvic floor, may be beneficial to women during labor. Available evidence suggests that dancing during the first stage of labor may decrease duration and intensity of pain and increase patient satisfaction, but further study is warranted. Ultimately, before assessing the implications of dance during labor, a feasibility study should be conducted to determine pregnant women's willingness to participate in a prospective or randomized controlled trial. (J Patient Cent Res Rev. 2020;7:213-217.)
\end{abstract}

Keywords $\quad$ pregnancy; first stage labor; exercise, dance; belly dancing; upright positioning; pain management

$\longrightarrow$ hildbirth is a natural phenomenon that has become increasingly medicalized in the United States. ${ }^{1}$ Pain reduction interventions during childbirth are primarily pharmacological, ${ }^{1}$ despite the psychological, emotional, spiritual, and pain management benefits associated with nonpharmacological approaches., ${ }^{2,3}$ Some pregnant women seek nonpharmacological interventions during labor in order to increase control of their labor and birth experience..$^{4-7}$ Health care providers recognize women's autonomy and seek to support women's goals for labor and childbirth. ${ }^{8}$

Light to moderate exercise for the duration of pregnancy has been well-researched and is generally recommended

Correspondence: Jessica J. F. Kram, MPH, Aurora Sinai Medical Center, 1020 N. 12th Street, \#4180, Milwaukee, WI 53233 (jessica.kram@aah.org) for healthy women. ${ }^{9}$ According to the American College of Obstetricians and Gynecologists (ACOG), exercising throughout pregnancy is likely beneficial and of minimal risk to most women with an uncomplicated pregnancy. ${ }^{9}$ Despite this, modifications to exercise regimens may be necessary given normal anatomic and physiologic changes in both women and the developing fetus. ${ }^{9}$ When women exercise throughout pregnancy, they have a decreased risk of gestational diabetes, preeclampsia, gestational weight gain, cesarean section, and operative vaginal delivery, in addition to decreased postpartum recovery time. ${ }^{9,10}$ Exercise during pregnancy also may increase cardiorespiratory fitness while promoting healthy blood flow, balance the neuroendocrine system, combat fatigue, and reduce stress. ${ }^{4,11}$

There is minimal evidence regarding exercise during the first stage of labor (ie, progressive dilation of cervix to $10 \mathrm{~cm}$ ) for pain reduction, progression of the first stage, patient satisfaction, etc. Anecdotally, health care 
providers often recommend going for a walk to help early latent labor progress. Some authors have written opinion pieces on their personal positive experiences with dancing during first stage of labor. ${ }^{12-15}$ Dance, a low-impact exercise in which pregnant women can participate throughout their pregnancy, ${ }^{10,11,16,17}$ could be used to help with the progression of labor. In this context, dance is a combination of pelvic movement, rhythmic body movement, upright positions, and position changes. ${ }^{6}$ For example, in some cultures specific types of dance, such as belly dance, are thought to facilitate labor and delivery. ${ }^{12}$ Moreover, belly dancing and other forms of dance throughout pregnancy may help to strengthen the muscles used during labor. ${ }^{10,15}$ Certain movements — such as expanding hip circles, which loosen and relax muscles of the pelvis and pelvic floor - may help to move the fetus down into the pelvis., ${ }^{6}, 12-14$ The term "dance" in this context is not literal, as it varies by woman, and women may only use some elements or movements associated with dance; rather, dance represents a range of body movements. ${ }^{13}$

Because spontaneous labor is associated with fewer interventions in labor, dance may be one way to reduce technological and medical interventions during labor while encouraging labor progression. ${ }^{2,18}$ Social media has increased interest in dancing during labor because of several circulating videos of pregnant women dancing during the final days of pregnancy and during latent-phase labor. ${ }^{19,20}$ Although beyond the scope of this topic synopsis, interest in dancing during labor has been impacted by social media, which warrants further investigation.

\section{Dance to Spur Upright Positioning and Mobility During Labor}

In many cultures, upright positioning during labor is common. ${ }^{21}$ Upright positioning and mobility is recommended because it likely improves maternal and fetal circulation while simultaneously improving maternal comfort. ${ }^{21-23}$ In upright positions, gravity may help the fetus descend though the pelvis while limiting compression of the abdominal blood vessels to maximize uterine blood flow to the placenta and fetus. ${ }^{6,13,22}$ Upright positions may reduce adverse effects associated with supine positioning, such as fetal heart rate decelerations, decreased uterine perfusion, and fewer uterine contractions. ${ }^{6,9}$ One study of healthy women found that upright and ambulatory positions shortened the duration of labor, increased likelihood of vaginal birth, decreased likelihood of operative birth, and decreased admission to the neonatal intensive care unit when compared to recumbent positioning. ${ }^{22}$ Women were less likely to have an epidural and had lower pain scores, although increased anxiety in nulliparous women was noted. ${ }^{22}$ In Western cultures, women are encouraged to lie down during labor, often to facilitate fetal monitoring; however, there is likely benefit to encouraging women to be upright and active to improve pain control and increase comfort in labor. ${ }^{18,22}$ Women's positions during first stage of labor may be as varied as the labors themselves. ${ }^{22}$ Low-impact dance may be one way to encourage the combination of both upright positioning and movement during the first stage of labor.

\section{Dance to Shorten Duration of Labor}

There have been conflicting study results regarding duration of the first stage of labor when women were encouraged to move and change positions. ${ }^{18}$ Of the studies reviewed, less than half identified that the length of labor was shortened when women participated in physical activity during the first stage. ${ }^{18}$ The authors concluded that further research was needed to evaluate the effects of exercise during the first stage of labor, particularly on labor duration. ${ }^{18}$

As mentioned, despite limited research, the use of gravity is believed to aid in the descent of the fetal head, leading to intensifying uterine contractions and increased labor progression. ${ }^{22}$ Although there have been mixed findings and few details regarding type, duration, and intensity of activity, positions, and mobility, ${ }^{18}$ it is possible to hypothesize that dancing and movements associated with dance could decrease the duration of the first stage of labor.

\section{Dance as a Form of Pain Management}

One study found that approximately $60 \%$ of primiparous women reported severe acute labor pain; even after analgesic drugs, about $50 \%$ of laboring women were not satisfied with the pain relief they received. ${ }^{24}$ This form of unrelieved pain is speculated to increase elective cesarean sections. ${ }^{2,25}$ Findings from another randomized control trial identified that dancing during the first stage of labor decreased pain when compared to controls, 
further supporting the use of upright position and pelvic movement. ${ }^{6}$ Significantly lower pain scores were noted among women in the dancing group 30 minutes and 60 minutes after intervention; however, the dancing group had lower pain scores prior to intervention, making a conclusion about dancing as a nonpharmacological intervention difficult. ${ }^{6}$ In another study, women reported less pain when standing for 30 minutes compared to lying supine or sitting. ${ }^{3}$ Further, one author noted that based on her personal experience of belly dancing during labor, the dance movements helped facilitate labor progress, decrease pain, and improve fetal oxygenation. ${ }^{12}$

Potentially, nonpharmacological pain management interventions such as dance, which are low in cost and free of serious adverse side effects, may provide women pain relief and feelings of control., 2,6,26

\section{Dancing May Improve Satisfaction}

Due to increased use of technological interventions, monitoring, and immobility during labor, some women experience increased fear, anxiety, loss of control, less autonomy or shared decision-making in labor, and possibly depression. ${ }^{1}$ They also may feel dissatisfaction with their labor experience. ${ }^{2,3,5,7,24,25,27,28}$ These negative emotions can have a lasting impact on laboring women. ${ }^{24}$ The Centers for Disease Control and Prevention claims that $9 \%$ of pregnant women and $10 \%$ of postpartum women meet the criteria for major depressive disorders. ${ }^{29}$ There is evidence that engaging in some form of creativity such as dance may lead to deep relaxation, with the potential to change attitude, emotional state, and perception of pain. ${ }^{28}$ Studies and opinion articles have described dancing during pregnancy and labor as joyful, relaxing, strengthening, and offering a connection between the mother and her developing baby. ${ }^{4,13,15,21,30,31}$

Some forms of dance, such as belly dancing, may positively affect body image and self-esteem. ${ }^{30}$ In the only randomized trial to evaluate dancing during the first stage of labor, women's satisfaction was significantly higher in the dancing group. ${ }^{6}$ However, this study also included other interventions, such as massage and the support of a partner, which makes results difficult to interpret. ${ }^{6}$ The impact of music alone has the potential to amplify the positive feedback associated with exercise, and music during labor has been found to decrease anxiety, pain, blood pressure, heart rate, and postpartum analgesic requirement. ${ }^{24}$ Because dancing usually occurs with music, these benefits may be intensified. Dancing to music chosen by the woman in labor may have a positive effect on her birthing experience.

Both ACOG and the American College of NurseMidwives support the use of nonpharmacological and nontechnological interventions during labor to improve comfort when feasible and safe. ${ }^{23,32}$ Improving women's comfort and freedom of mobility during labor also may improve overall satisfaction.

\section{Other Considerations}

Maternal and fetal implications of dancing during the first stage of labor are relatively unknown, although it is speculated to have benefit without harm. Lowimpact exercise and its effects on pregnancy have been studied and is recommended by ACOG; dance likely fits into this category. ${ }^{9}$ One review found that women who participated in aerobic dance and strength twice weekly for a minimum of 12 weeks did not have an increased risk of low-birth-weight infants, preterm birth, or low Apgar scores. ${ }^{10}$ When comparing lowimpact dance with walking during pregnancy, the maternal heart rate to $\mathrm{VO}_{2}$ (oxygen uptake) relationship could increase, which may theoretically increase the possibility of fetal tachycardia. ${ }^{9,16,17}$ Additionally, passive swinging associated with dancing during pregnancy could potentially increase the heart rate of the fetus as well as fetal movement. ${ }^{33}$ Because there have been controversial findings regarding the effect of exercise on fetal cardiac function during pregnancy, ${ }^{34}$ the potential for fetal stress during the implementation of dancing during labor should be acknowledged. ${ }^{34}$

Interestingly, along with potential unknown physical implications, there is a potential role in the development of the fetus's future personality and talents. ${ }^{33} \mathrm{~A}$ survey study of professional dancers identified that mothers who danced throughout their pregnancy were more likely to have children who needed to be rocked to sleep during the first year of life and were more likely to play a musical instrument. ${ }^{33}$

\section{Conclusions}

The type of dance and the benefits of dancing during pregnancy and labor have been understudied. Previous 
research has shown that women's sense of control during birth is the main contributing factor to birth satisfaction and emotional well-being, which might be improved with dancing. ${ }^{4,15,21,25,30,31}$ However, because lying down facilitates fetal monitoring during labor, this seemingly simple intervention represents a major birth culture shift in U.S. hospitals and may prove difficult to implement. ${ }^{18,27}$ Considerations include staff training for effective delivery of this particular intervention, measuring the start of intervention, standardized reporting for types of movements, maintaining the appropriate amount of monitoring of labor progress, music selection, noise-level monitoring, and more. ${ }^{27}$

Before a larger prospective trial can ensue, a feasibility study should be conducted to determine pregnant women's willingness to participate in a future study aimed at evaluating low-impact dance as a potential form of pain reduction during the first stage of labor. A feasibility study also could help evaluate pregnant women's perceptions and opinions about this potential form of pain reduction. Understanding women's attitudes regarding both dance during labor and study participation can inform a randomized control trial. Such a study would provide more insight into the potential benefits of dancing during the first stage of labor as it relates to labor progression, pain reduction, and satisfaction.

\section{Patient-Friendly Recap}

-While exercise during pregnancy has been found to positively affect both mother and fetus, little is known about the effects of lowimpact dance in the first stage of labor.

- After reviewing existing literature, the authors found some evidence that low-impact dancing may decrease pain and duration of labor while increasing women's satisfaction with the labor experience.

- Prior to designing a large randomized trial, the authors recommend conducting a feasibility study to elicit pregnant women's attitudes toward dancing during labor as well as their willingness to participate in a research study at that time.

\section{Author Contributions}

Study design: all authors. Data acquisition or analysis: all authors. Manuscript drafting: all authors. Critical revision: all authors.

\section{Conflicts of Interest}

None.

\section{References}

1. Johanson R, Newburn M, Macfarlane A. Has the medicalisation of childbirth gone too far? BMJ. 2002;324:892-5. CrossRef

2. Abdul-Sattar Khudhur Ali S, Mirkhan Ahmed H. Effect of change in position and back massage on pain perception during first stage of labor. Pain Manag Nurs. 2018;19:288-94. CrossRef

3. Simkin P, Bolding A. Update on nonpharmacologic approaches to relieve labor pain and prevent suffering. J Midwifery Womens Health. 2004;49:489-504. CrossRef

4. Campbell L. Movement and dance: deep medicine for pregnancy, labour and birth. Essentially MIDIRS. 2013;4(4):40-5.

5. Leavitt JW. Dancing through labor \& delivery: the passion of Sheila Kitzinger. Feminist Collections: A Quarterly of Women's Studies Resources. 2016;37(3):5-7.

6. Abdolahian S, Ghavi F, Abdollahifard S, Sheikhan F. Effect of dance labor on the management of active phase labor pain \& clients' satisfaction: a randomized controlled trial study. Glob J Health Sci. 2014;6:219-26. CrossRef

7. Meyer S. Control in childbirth: a concept analysis and synthesis. J Adv Nurs. 2013;69:218-28. CrossRef

8. Kotaska A. Informed consent and refusal in obstetrics: a practical ethical guide. Birth. 2017;44:195-9. CrossRef

9. ACOG Committee Opinion No. 650: Physical activity and exercise during pregnancy and the postpartum period. Obstet Gynecol. 2015;126:e135-42. CrossRef

10. Nascimento SL, Surita FG, Cecatti JG. Physical exercise during pregnancy: a systematic review. Curr Opin Obstet Gynecol. 2012;24:387-94. CrossRef

11. Halvorsen S, Haakstad LA, Edvardsen E, Bø K. Effect of aerobic dance on cardiorespiratory fitness in pregnant women: a randomised controlled trial. Physiotherapy. 2013;99:42-8. CrossRef

12. Moore C. Belly dance \& birth. Midwifery Today Int Midwife. 2005;(73):28-9.

13. Oeftering GM. Belly-dancing through pregnancy: a way to give birth and not be delivered. Int J Childbirth Educ. 1999;14(3):6-8.

14. Moyer K. Media reviews: Dance of the Womb. Midwifery Today Int Midwife. 2010;(95):59.

15. MacGregor HE, Los Angeles Times. The belly dances, the muscles follow. Published 2002 Nov 18. https://www.latimes. com/archives/la-xpm-2002-nov-18-he-fitness 18-story.html. Accessed March 18, 2019.

16. McMurray RG, Katz VL, Poe MP, Hackney AC. Maternal and fetal responses to low-impact aerobic dance. Am J Perinatol. 1995;12:282-5. $\underline{\text { CrossRef }}$

17. McMurray RG, Hackney AC, Guion WK, Katz VL. Metabolic and hormonal responses to low-impact aerobic dance during pregnancy. Med Sci Sports Exerc. 1996;28:41-6. CrossRef

18. Hollins Martin CJ, Martin CR. A narrative review of maternal physical activity during labour and its effects upon length of first stage. Complement Ther Clin Pract. 2013;19:44-9. CrossRef 
19. Michallon C, DailyMail.com. Getting in the family groove! Woman who is pregnant with triplets tries to dance her way into labor, shaking her enormous baby bump while boogying with her husband and their daughter. Published 2018 Oct 22. https://www.dailymail.co.uk/femail/article-6303595/Heavilypregnant-woman-expecting-TRIPLETS-tries-dance-waylabor.html. Accessed October 15, 2019.

20. Baulkman J, DailyMail.com. Doctor helps women through labor by dancing with them in hilarious videos - and research shows it does help. Published 2018 Jan 19. https://www. dailymail.co.uk/health/article-5289287/Doctor-helps-womenlabor-dancing-them.html. Accessed October 15, 2019.

21. Henley-Einion A. The ecstasy of the spirit: five rhythms for healing. Pract Midwife. 2007;10:20, 22-3.

22. Lawrence A. Lewis L, Hofmeyr GJ, Styles C. Maternal positions and mobility during first stage labour. Cochrane Database Syst Rev. 2013;(10):CD003934. CrossRef

23. ACOG Committee Opinion No. 766: Approaches to limit intervention during labor and birth. Obstet Gynecol. 2019;133:e164-73. CrossRef

24. Simavli S, Gumus I, Kaygusuz I, Yildirim M, Usluogullari B, Kafali H. Effect of music on labor pain relief, anxiety level and postpartum analgesic requirement: a randomized controlled clinical trial. Gynecol Obstet Invest. 2014;78:244-50. CrossRef

25. Buitendijk S. Gender issues in determining the service and research agenda for pregnancy and birth care: the case of home birth in the Netherlands. Interdiscip Sci Rev. 2011;36:193-202. CrossRef

26. Simkin PP, M. O'Hara M. Nonpharmacologic relief of pain during labor: systematic reviews of five methods. Am J Obstet Gynecol. 2002;186(5 Suppl Nature):S131-59. CrossRef
27. Kimber L, McNabb M, Mc Court C, Haines A, Brocklehurst P. Massage or music for pain relief in labour: a pilot randomised placebo controlled trial. Eur J Pain. 2008;12:961-9. CrossRef

28. Demecs IP, Fenwick J, Gamble J. Women's experiences of attending a creative arts program during their pregnancy. Women Birth. 2011;24:112-21. CrossRef

29. ACOG Committee Opinion No. 757: Screening for perinatal depression. Obstet Gynecol. 2018;132:e208-12. CrossRef

30. Jackson I. Empowering embodiment through belly dancing in pregnancy: a phenomenological exploration. Pract Midwife. 2015;18(5):27-30.

31. Sanders SG. Dancing through pregnancy: activity guidelines for professional and recreational dancers. J Dance Med Sci. 2008;12:17-22.

32. American College of Nurse-Midwives. Position statement: appropriate use of technology in childbirth. Updated 2014 May. https://www.midwife.org/acnm/files/ACNMLibraryData/ UPLOADFILENAME/000000000054/Appropriate-Use-ofTechnology-in-Childbirth-May-2014.pdf. Accessed October 15, 2019.

33. Bellieni CV, Cordelli DM, Bagnoli F, Buonocore G. 11to 15 -year-old children of women who danced during their pregnancy. Biol Neonate. 2004;86:63-5. CrossRef

34. Brik M, Fernández-Buhigas I, Martin-Arias A, Vargas-Terrones M, Barakat R, Santacruz B. Does exercise during pregnancy impact on maternal weight gain and fetal cardiac function? A randomized controlled trial. Ultrasound Obstet Gynecol. 2019;53:583-9. CrossRef

(C) 2020 Aurora Health Care, Inc. 